\title{
Nuances of the psychogastroenterology patient: A predictive model for gastrointestinal quality of life improvement
}

\author{
Megan E. Riehl (1) | Jami A. Kinnucan | William D. Chey | Ryan W. Stidham
}

Division of Gastroenterology, University of Michigan, Ann Arbor, Michigan, USA

\section{Correspondence}

Megan E. Riehl, Division of

Gastroenterology, University of Michigan, 3912 Taubman Center, 1500 E. Medical Center Drive, Ann Arbor, 48109-5362 MI, USA.

Email: mriehl@med.umich.edu

\begin{abstract}
Background: Gastrointestinal conditions are multifactorial in nature, and certain patients can benefit greatly from brain-gut psychotherapies delivered by mental health professionals who specialize in psychogastroenterology. This study aimed to identify features associated with improvements in GI-specific quality of life scores following behavioral health interventions (BHI). The second aim was to create a psychogastroenterology referral care pathway incorporating identified characteristics for greatest benefit from $\mathrm{Gl}$-specific behavioral therapy.

Methods: We performed a prospective observational study of 101 (63 women; median age, 45 years) gastroenterology patients referred for psychogastroenterology consultation at a single center. Patients attended an average of seven sessions with a single GI psychologist where evidence-based brain-gut psychotherapies were employed. Gl-specific quality of life (IBS-QOL) and psychological distress (BSI-18) were assessed before and after BHI. Patients completed self-reported questionnaires. We performed a multivariable analysis to determine predictors associated with IBS-QOL score improvement. Key Results: A total of 53 (52.5\%) patients experienced improvement in IBS-QOL score. Patients with improved IBS-QOL scores had significantly higher baseline BSI general domain $\mathrm{T}$-scores (61.9 vs. 56.9, $\mathrm{P}=0.002$ ). Female gender (odds ratio [OR], 3.2), pretreatment $\mathrm{BSI}$ somatization $\mathrm{T}$-score $\geq 63$ (OR, 3.7), and a diagnosis of depression (OR, 4.2) were associated with greater odds of IBS-QOL score improvement following BHI.

Conclusions and Inferences: We identified factors associated with response to GIspecific $\mathrm{BHI}$ to aid in optimizing the utilization of psychogastroenterology services and provide referring providers with information to inform treatment recommendations. Female patients with disorders of gut-brain interaction (DGBIs), high somatization, and depression should be considered a priority for brain-gut psychotherapies.
\end{abstract}

\section{KEYWORDS}

cognitive behavioral therapy, disorders of gut-brain interaction, gut-directed hypnotherapy, inflammatory bowel disease, psychogastroenterology 


\section{1 | INTRODUCTION}

In patients with disorders of gut-brain interaction (DGBIs), comorbid depression and anxiety disorders occur in approximately $30 \%$ and $50 \%$ of patients, respectively. ${ }^{1}$ Crohn's disease and ulcerative colitis have a similar prevalence of mood disorders compared with those with DGBIs. ${ }^{2}$ Brain-gut psychotherapies are effective for improving quality of life and disease experience for a wide range of $\mathrm{Gl}$ conditions targeting the multifactorial nature of DGBIs, ${ }^{3,4}$ upper $\mathrm{Gl}$ conditions ${ }^{5}$ (eg, heartburn, dysphagia, and globus), and inflammatory bowel disease (IBD). ${ }^{2,6}$ As a result, "psychogastroenterology" has organically emerged as an effective treatment modality necessary for holistic GI care. ${ }^{7}$

At present, access to integrated psychogastroenterology providers remains limited. While enthusiasm for referrals makes it clear that gastroenterologists and patients recognize the value of psychogastroenterology services, the patient phenotype most likely to benefit from these services has not been fully elucidated. As individualized care pathways emerge and behavioral health is incorporated into treatment algorithms, both medical specialists and patients will benefit from clear guidance regarding the best psychologic resource to be used at a given time.

Analyses were performed at the group level in gastroenterology patients with DGBIs. We aimed to identify features associated with improvements in psychologic function and Gl-specific quality of life (QOL) using validated measures, as well as patient-reported mental health improvements following behavioral health interventions.

\section{2 | METHODS}

\section{1 | Study population}

Patients seen in our university-based outpatient gastroenterology clinic were referred for $\mathrm{GI}$ Behavioral Health Intervention (BHI) utilizing our $\mathrm{BHI}$ referral criteria: patients with DGBls, those lacking severe psychiatric comorbidity, those with insight into the role of stress on their Gl functioning, and those motivated to address their GI symptoms using brain-gut psychotherapy. Exclusion criteria were untreated moderate-to-severe psychiatric comorbidity and poor insight or motivation. Patients presented for the management of DGBls; however, some patients had a relevant co-diagnosis of IBD, chronic pain conditions, and upper GI complaints. Referred patients were consecutively approached for participation in the study; those providing informed consent completed validated questionnaires prior to and at the completion of $\mathrm{BHI}$ (Appendix S1). Patient self-reported medical and prescription data were augmented and verified by review of electronic medical records.

\section{$2.2 \mid$ Measures}

\subsection{1 | Psychosocial checklist}

Patients were asked to review a list of 36 current or past psychosocial stressors and identify which they have experienced. Key psychosocial stressors listed include the following: work problems,

\section{Key Points}

- Gastrointestinal conditions are multifactorial in nature, and certain patients can benefit greatly from brain-gut psychotherapies delivered by mental health professionals who specialize in psychogastroenterology.

- Females with disorders of gut-brain interaction, high somatization, and depression should be considered priority for brain-gut psychotherapies. Behavioral health outcomes were not limited to disease; patients with IBD should be routinely considered for referral.

- Optimizing utilization of Gl-specific behavioral health specialists for the best outcomes can maximize quality of life and disease experience, but also improve valuebased care.

caregiver stress, difficulties with communication, loss of a loved one, anxiety, nightmares, sexual dysfunction, chronic pain, thoughts of self-harm, abuse/trauma, eating disorder, and addiction.

\subsection{2 | Demographic and clinical information}

Patients provide their name, age, occupation, highest level of education completed, weight, height, and type of GI diagnosis.

\subsection{3 | Concomitant treatment form}

Patient is asked to list medical and/or psychiatric conditions and they currently have including psychiatric diagnoses and treatment history. Chronic pain conditions include chronic migraines or headaches, fibromyalgia, temporomandibular joint syndrome (TMJ), and interstitial cystitis (IC). The use of psychoactive prescription medications includes tricyclic antidepressants and atypical antipsychotic agents. Opioids, benzodiazepines, and illicit substance used for the 12 months preceding $\mathrm{BHI}$ referral were also collected.

\subsection{4 | Rating of symptom severity}

Patients self-reported the severity of their last GI symptoms flareup on a 10-point Likert scale with higher scores indicating greater symptom severity.

\subsection{5 | Irritable Bowel syndrome quality of life questionnaire (IBS-QOL)}

This is a 34-item self-report instrument that measures health-related QOL with eight symptom dimensions on a five-point response scale: dysphoria, health worry, social reaction, interference with activity, sexual impact, body image, and relationships. ${ }^{8}$ Items are summed and averaged for a total score which is transformed to a 0-100 scale 
with higher scores indicating better IBS-specific QOL. Clinically, significant QOL improvement is defined as an increase of 14 or more points. ${ }^{?}$

\subsection{6 | Brief symptom inventory-18 (BSI-18)}

Is an 18-item reliable measure of general psychological distress in medical populations with four domains graded on a five-point Likert scale including: somatization (the psychological tendency to experience a multitude of non-specific body symptoms), depression, anxiety, and Global Severity Index (GSI). ${ }^{10}$ Subscale scores range from 0 to 72 and are converted to T-scores. A BSI T-score $\geq 63$, which is greater than the 90th percentile of the reference population, is considered significantly distressed. ${ }^{11}$ A T-score $<63$ is within the normal range.

\subsection{7 | Psychosocial clinical interview}

All patients completed the initial psychological evaluation by a single GI psychologist (MER) to further determine whether behavioral health treatment is appropriate for their current medical complaints and to assess whether a psychiatric comorbidity takes precedence. Insight into the manner in which psychological factors can impact the GI symptom experience, in addition to patient's motivation to engage in $\mathrm{BHI}$ was assessed. At the conclusion of the interview, appropriate patients were provided with a treatment plan with a target of seven sessions.

\subsection{Behavioral health intervention}

Given that there is not a single standardized protocol for using CBT for gastrointestinal symptoms, our study consistently incorporated the application of CBT and gut-directed hypnotherapy interventions which were appropriate for patients presenting complaints. ${ }^{3}$ Patient progress or willingness to proceed impacted the total number of sessions.

\subsection{1 | Cognitive behavioral therapy (CBT)}

This approach is routinely tailored to patients with $\mathrm{Gl}$ conditions by providing psychoeducation about the body's stress response and how it can impact gastrointestinal functioning, address healthspecific mood symptoms, improve coping skills and increase medical adherence. ${ }^{4,6,12}$ Learning how to reduce physiological arousal and attenuate hypervigilance through the practice of relaxation skills (eg, diaphragmatic breathing, muscle relaxation, self-hypnosis) augments the patient's capability to maximally participate in CBT exercises.

\subsection{2 | Gut-directed hypnotherapy}

This intervention has several evidence-based benefits, specifically targeting the down-regulation of unpleasant GI sensations by normalizing pain processing and perception via the brain-gut axis. It is successfully used in patients with functional abdominal and bowel complaints to improve health outcomes of visceral sensitivity, gut motility, central processing, and overall psychological status. ${ }^{13-15}$ This study used the North Carolina Protocol, a seven-session scripted protocol designed for patients with IBS or IBD. ${ }^{13,16,17}$ In patients with comorbid upper GI complaints, appropriate modifications were made utilizing tailored hypnotic suggestions. ${ }^{18}$

\subsection{Outcome assessment}

Our primary outcome assessment was improved IBS-QOL score post-BHI. A clinically significant improvement in Gl-specific QOL was defined as an increase in IBS-QOL score $\geq 14$ points post- $\mathrm{BHI}$ treatment. ${ }^{9}$ At termination of $\mathrm{BH}$ treatment, participants also completed BSI survey and self-reported patient outcome measures including the following: perceived degree of improvement in $\mathrm{GI}$ symptom experience (excellent, moderate, slight, no improvement, and worse), reduction in on-demand medication use for symptom relief and the use of $\mathrm{BHI}$ skills at the completion of therapy (CBT, diaphragmatic breathing, and self-hypnosis).

\section{5 | Statistical analysis}

Demographic, clinical, and survey reply features prior to the start of $\mathrm{BHI}$ were compared between patients who experienced an improvement in IBS-QOL of 14 or more points and those without an IBS-QOL improvement. Univariate analysis was performed using student's $t$ test, chi-squared test, or Fisher's exact test in the setting of low-frequency categorical events. Multivariable logistic regression model building utilized a backward variable selection process with forced inclusion of age, gender, and IBD deemed relevant a priori. Continuous variables were also explored as categorical variables (with and without ordinal features) to provide the best model fit. Analysis of maximum likelihood estimates provided hazard ratios and confidence limits for each parameter within the model. Univariate analyses considered a $P$-value of $\leq 0.01$ as statistically significant after applying Bonferroni correction to control for Type 1 error due to multiple comparisons; regression analyses retained a statistical significance of $P<0.05$. All statistical analyses were conducted using SAS 9.4 (Cary, NC).

\section{3 | RESULTS}

\section{1 | Patient characteristics}

A total of 101 patients with DGBIs enrolled for prospective observation while undergoing $\mathrm{BHI}$ from 2015 to 2018 . The overall population was $62.4 \%$ female, with $27.7 \%$ having comorbid depression, $42.6 \%$ had comorbid anxiety, $22.8 \%$ had underlying IBD, and $22.8 \%$ had current psychotropic therapy use. The overall mean age was 45.1 years with a range of 18.1-80.4 years of age, and no difference in age was observed by QOL score improvement. The population baseline BSI general score was 53.6 (SD 7.9), and baseline IBS-QOL score was 54.0 (SD 16.5). Fifty-three patients (52.3\%) experienced 
TABLE 1 Patient characteristics

\begin{tabular}{|c|c|c|c|}
\hline & \multicolumn{3}{|c|}{ Postintervention QOL Change } \\
\hline & \multirow{2}{*}{$\begin{array}{l}\text { No improvement } \\
\mathrm{n}=48\end{array}$} & \multirow{2}{*}{$\frac{\text { Improvement }}{n=53}$} & \multirow[t]{2}{*}{$P$} \\
\hline & & & \\
\hline Age, years (SD) & $47.4(16.5)$ & $42.9(15.3)$ & 0.181 \\
\hline Gender, female (\%) & $24(50)$ & $39(73.6)$ & 0.018 \\
\hline $\begin{array}{l}\text { Lower tract symp- } \\
\text { toms (\%) }\end{array}$ & $40(83)$ & $50(92.4)$ & 0.112 \\
\hline $\begin{array}{l}\text { Upper tract symp- } \\
\text { toms (\%) }\end{array}$ & 15 (31.9) & $5(9.8)$ & 0.007 \\
\hline \multicolumn{4}{|l|}{ Co-diagnoses } \\
\hline $\begin{array}{l}\text { IBD co-diagnosis } \\
(\%)\end{array}$ & $9(18.8)$ & $13(24.5)$ & 0.482 \\
\hline Depression (\%) & $8(16.7)$ & $20(37.7)$ & 0.018 \\
\hline Anxiety (\%) & $20(41.7)$ & $23(47.7)$ & 0.578 \\
\hline Migraine (\%) & $7(14.6)$ & $13(24.5)$ & 0.210 \\
\hline Fibromyalgia (\%) & $6(12.5)$ & 9 (17.0) & 0.527 \\
\hline $\begin{array}{l}\text { Intersticial cysti- } \\
\text { tis (\%) }\end{array}$ & $7(14.6)$ & $8(15.1)$ & 0.943 \\
\hline TMJ (\%) & $6(12.5)$ & $10(18.9)$ & 0.381 \\
\hline \multicolumn{4}{|l|}{ Medication use } \\
\hline $\begin{array}{l}\text { Benzodiazepine } \\
\text { use } \mathrm{Hx}(\%)\end{array}$ & $5(10.6)$ & $6(11.8)$ & 0.860 \\
\hline $\begin{array}{l}\text { Narcotic use } \mathrm{Hx} \\
\text { (\%) }\end{array}$ & $1(2.1)$ & $5(9.8)$ & 0.113 \\
\hline $\begin{array}{l}\text { Psychotropic } \\
\text { use (\%) }\end{array}$ & $12(25.5)$ & $11(21.6)$ & 0.644 \\
\hline \multicolumn{4}{|c|}{ Patient-reported symptoms } \\
\hline $\begin{array}{l}\text { Psychosocial } \\
\text { stressors (SD) }\end{array}$ & $3.2(0.7)$ & $3.5(0.7)$ & 0.190 \\
\hline $\begin{array}{l}\text { GI Sx severity } \\
\text { rating (SD) }\end{array}$ & $7.2(1.6)$ & $7.7(1.6)$ & 0.151 \\
\hline
\end{tabular}

a clinically meaningful improvement in IBS-QOL scores. Separating the overall study population by QOL improvement following BSI, positive predictors for clinically meaningful improvement in IBSQOL scores were a higher baseline BSI score $(P=0.003)$ and the absence of upper tract symptoms $(P=0.007$, Table 1$)$. Males comprised $37.6 \%$ of the cohort and male vs female sex and exhibited a non-significant trend of non-response to $\mathrm{BHI}(63.3 \%$ vs. $38.1 \%$, $P=0.018$ ). The presence of upper tract $\mathrm{Gl}$ symptom complaints occurred in 20 study subjects and was associated with non-response to $\mathrm{BHI}(31.2 \%$ vs. $9.4 \%, P=0.007) .21$ of 101 patients had IBD, 10 with Crohn's disease, and 11 with ulcerative colitis. The presence of IBD was not associated with achieving a clinically meaningful improvement in IBS-QOL scores $(P=0.482)$. Co-existing chronic pain conditions were also not associated with responsiveness to $\mathrm{BHI}$ $(P=0.673)$. Use of psychoactive medications, benzodiazepines, or opioids within the prior year was not associated with $\mathrm{BHI}$ response $(P=0.714)$. No report of suicidal ideation and formal eating disorder diagnoses occurred in this cohort.
TAB LE 2 Brief symptom inventory scores before and after behavioral health interventions

\begin{tabular}{|c|c|c|c|c|c|}
\hline & \multirow{2}{*}{\multicolumn{2}{|c|}{$\begin{array}{l}\begin{array}{l}\text { No } \\
\text { Improvement }\end{array} \\
n=48\end{array}$}} & \multirow{2}{*}{\multicolumn{2}{|c|}{$\begin{array}{l}\text { Improvement } \\
\mathrm{n}=53\end{array}$}} & \multirow[b]{3}{*}{$P$} \\
\hline & & & & & \\
\hline & Value & SD & Value & SD & \\
\hline $\begin{array}{l}\text { BSI-GSI PreTx score } \\
\text { (Raw) }\end{array}$ & 13.7 & 9.7 & 20.5 & 12.0 & 0.003 \\
\hline BSI-GSI PreTx ZScore & 57.0 & 8.5 & 61.9 & 8.7 & 0.006 \\
\hline BSI-GSI PostTx score & 53.3 & 8.1 & 53.3 & 7.9 & 0.966 \\
\hline BSI-GSI Score change & 3.4 & 7.1 & 8.6 & 7.4 & 0.001 \\
\hline $\begin{array}{l}\text { BSI-Dep PreTx score } \\
\text { (Raw) }\end{array}$ & 4.6 & 4.5 & 6.8 & 5.2 & 0.024 \\
\hline BSI-Dep PreTx ZScore & 54.9 & 9.6 & 59.4 & 9.8 & 0.023 \\
\hline BSI-Dep PostTx score & 51.9 & 8.5 & 51.0 & 8.4 & 0.605 \\
\hline BSI-Dep Score change & 57.0 & 8.5 & 61.9 & 8.7 & 0.006 \\
\hline $\begin{array}{l}\text { BSI-ANX PreTx score } \\
\text { (Raw) }\end{array}$ & 4.8 & 3.4 & 6.5 & 4.7 & 0.039 \\
\hline BSI-ANX PreTx Zscore & 55.1 & 9.0 & 58.2 & 9.9 & 0.102 \\
\hline BSI-ANX PostTx score & 51.6 & 7.4 & 51.1 & 7.5 & 0.741 \\
\hline $\begin{array}{l}\text { BSI-ANX Score } \\
\text { change }\end{array}$ & 3.4 & 7.1 & 7.1 & 7.7 & 0.015 \\
\hline $\begin{array}{l}\text { BSI-SOM PreTx score } \\
\text { (Raw) }\end{array}$ & 4.4 & 3.4 & 7.2 & 4.7 & 0.001 \\
\hline $\begin{array}{l}\text { BSI-SOM PreTx } \\
\text { Zscore }\end{array}$ & 56.8 & 9.0 & 62.5 & 9.3 & 0.003 \\
\hline BSI-SOM PostTx score & 53.5 & 9.0 & 55.1 & 8.2 & 0.349 \\
\hline $\begin{array}{l}\text { BSI-SOM Score } \\
\text { change }\end{array}$ & 3.2 & 7.6 & 7.5 & 7.5 & 0.005 \\
\hline
\end{tabular}

Abbreviations: ANX: Anxiety Subscore; DEP: Depression subscore; GSI: General Severity Index; SOM: Somatization subscore.

\subsection{Pre-BH intervention BSI scores and association with IBS-QOL score improvement}

Those with clinically meaningful improvements in IBS-QOL scores had significantly higher baseline BSI general domain T-scores (61.9 vs. $56.9, P=0.002)$. Higher pretreatment BSI somatization T-scores $(P<0.001)$ were associated with an improvement in IBS-QOL score (Table 2). Unsurprisingly, following $\mathrm{BHI}$, anxiety $(P=0.015)$, depression $(P=0.005)$, and somatization $(P<0.001)$ BSI subscores all improved relative to baseline values, defined as a T-Score $\geq 63$.

\section{3 | Multivariable model for predicting QOL improvement following $\mathrm{BH}$ intervention}

Adjusted analysis identified several pretreatment predictors of IBSQOL score improvement following BHI (Table 3). Females were more than three times as likely as males to experience clinically meaningful improvements in IBS-QOL score. Those with a pretreatment BSI somatization T-score of 63 or greater (upper quartile of the cohort) had a 3.7-fold greater odds of experiencing a clinically meaningful 
TABLE 3 Multivariable model of achieving quality of life improvement following behavioral health intervention

\begin{tabular}{|lllrl|}
\hline Variable & Odds Ratio & $\begin{array}{l}\text { 95\% Confidence } \\
\text { Limits }\end{array}$ & \multicolumn{1}{l}{ P } \\
\hline Age & 0.98 & 0.95 & 1.01 & 0.183 \\
\hline Gender, Female & 3.25 & 1.13 & 9.35 & 0.029 \\
\hline BSI SOM >63 & 3.74 & 1.19 & 11.72 & 0.024 \\
\hline Diagnosis major depression & 4.20 & 1.22 & 14.47 & 0.023 \\
\hline Diagnosis anxiety disorder & 0.37 & 0.11 & 1.18 & 0.092 \\
\hline Absence of upper GI functional & 6.48 & 1.70 & 24.74 & 0.006 \\
\hline symptoms & & & & \\
\hline IBD co-diagnosis & 0.96 & 0.31 & 3.00 & 0.942 \\
\hline
\end{tabular}

Bold indicates significant values. improvement in IBS-QOL score following BHI. Those with a pretreatment BSI general score of 63 or greater also had greater odds of successful response to $\mathrm{BHI}(\mathrm{OR} 2.3,95 \% \mathrm{Cl} 1.2,4.1)$, but this was not retained in the final model due to co-linearity with the BSI-somatization. A diagnosis of depression (OR 4.20, 95\% Cl 1.22, 14.47) but not anxiety was associated with greater odds of meaningful IBS-QOL score improvement following BHI. Psychoactive medication, narcotic, nor benzodiazepine were risk factors for failure of $\mathrm{BHI}$. The diagnosis of IBD ( $P=0.942)$ or chronic pain conditions $(P=0.869)$ was not risk factors for $\mathrm{BHI}$ failure. Finally, the presence of upper tract symptoms (which could co-occur with lower tract symptom complaints) significantly reduced the odds of experiencing a meaningful improvement in IBS-QOL score (OR 0.15, 95\% $\mathrm{Cl} 0.04,0.59)$ on adjusted analysis.

\section{4 | Patient-reported improvements and self- directed use of behavioral health techniques}

The vast majority of patients reported continued use of behavioral health skills, with $91 \%$ indicating continued diaphragmatic breathing use and $96 \%$ reporting continued CBT skill set use at the completion of their therapy course. Those patients who had an improved IBS-QOL score reported reduced use of on-demand medications for $\mathrm{Gl}$ symptom relief (66.0\% vs. $34.0 \%, P=0.007)$. Additionally, subjective patient self-report of improvement following $\mathrm{BHI}$ demonstrated poor agreement with objective IBS-QOL scores (=0.26). Overall, of the 45/101 patients self-reporting moderate or better improvement following $\mathrm{BHI}$, only $37.8 \%(17 / 45)$ demonstrated a 14 point or greater improvement on the IBS-QOL instrument $(P=0.008)$ (Figure 1$)$.

\section{4 | DISCUSSION}

In summary, we identify several factors associated with GI behavioral health interventions that successfully improve GI-specific QOL scores. Female patients with high somatization scores appear to have the most potential for improvement following BHI for FGIDs. Alternatively, male patients and those presenting with co-occurring upper GI tract functional symptoms appear to be less responsive to BHI. Finally, a co-diagnosis of IBD, a history of opioid, benzodiazepine, or psychoactive medication use does not appear to impact success of BHI. Understanding these features may help providers encourage patients with a high probability of response to undergo $\mathrm{BHI}$.

The reasons for difference in gender response to $\mathrm{BHI}$ remain unclear and could be linked to fundamental aspects of bowel symptom experience that are uncaptured by existing measures of therapeutic response and QOL. Further evidence of shortcomings in understanding treatment response may be linked to limitations in how we measure response. In our study, despite the IBS-QOL being an established measure, a bidirectional discrepancy was present between patients' subjective perception of improvement and their IBS-QOL score improvement. This suggests that patient perceptions of QOL and therapeutic benefit, at least in the context of $\mathrm{GI} \mathrm{BHI}$, are impacted by unmeasured variables. One consideration of this discrepancy is that some patients consciously or unconsciously may have been skewed toward more positive responses given they were returning treatment completion forms to the office of the single provider. While a limitation, it is a reality of this objective study.

Prior work has demonstrated that upper GI tract digestive complaints are more resistant to $\mathrm{BHI} .{ }^{5,19}$ While our analysis found them less likely to respond to $\mathrm{BHI}$, this indicates comorbid bowel and esophageal complaints likely require more intensive therapy and further customized $\mathrm{BHI}$ regimens.

Not surprisingly, patients reporting clinically significant somatization were found to be highly successful in behavioral therapy. We must also acknowledge that in a GI patient population, the BSI somatization score may be a measure of GI symptom severity; therefore, we may anticipate those patients with the worst Gl symptoms at baseline and may be more likely to respond to $\mathrm{BHI}$. This supports previous reports where hypnotherapy was superior to other intervention for reducing bothersome non-GI symptoms in DGBIs. ${ }^{20-22}$

These results should be interpreted in the context of several limitations. Firstly, we used the IBS-QOL, which is a QOL measurement tool validated in IBS patients, as our primary measure of improvement of DGBIs following BHI. This tool has not been validated in IBD or upper tract symptoms, and as such, the instrument conceivably may perform differently in patients with non-IBS diagnoses. While we acknowledge a lack of patient characterization, all referred patients had functional bowel complaints as the primary reason for referral and we elected to use a single instrument to minimize patient reporting burden. Secondly, while all patients received gut-directed 


\section{PATIENT QOL IMPROVEMENT $(N=101)$}

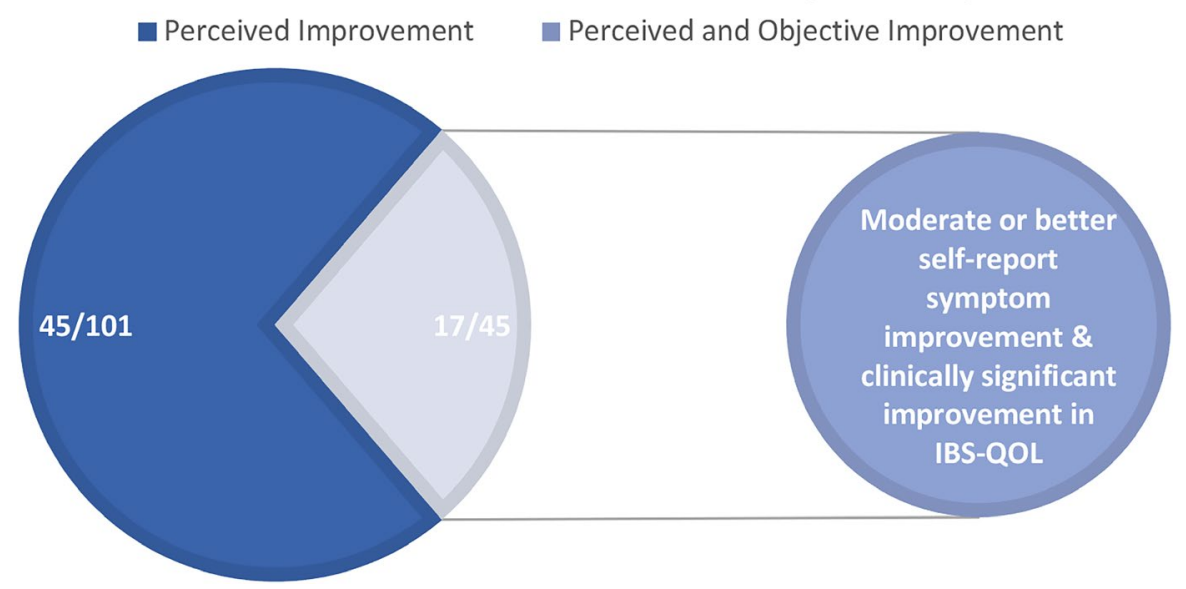

FIGURE 1 Patient quality of life improvement following behavioral health intervention hypnotherapy and CBT interventions, the lack of standardized treatment for every patient introduced an uncontrolled variable that this study was not designed to evaluate and should be part of future controlled interventional studies. Further, therapy was administered by a single GI behavioral health psychologist whose individual characteristics administrating care could influence results. However, this limitation also underscores the importance of training GI psychologists as there is a current shortage of available providers. Finally, over the course of the study, referring gastroenterologists' likely improved their patient selection for $\mathrm{BHI}$ referral as they received both psychologist and patient feedback. While potentially impacting results, if gastroenterologist referral pattern changes improved overall patients' BHI success, this would demonstrate the potential for improving patient selection practices. While the limitations highlighted preclude a conclusion of $\mathrm{BHI}$ effectiveness for improving functional bowel symptoms in patients with important co-diagnoses, the results support investment in more rigorous studies.
Our findings are consistent with other investigations of behavioral health utilization in gastroenterology. We propose a preliminary schema to aid gastroenterology providers in identifying patients for $\mathrm{BH}$ ls with a higher likelihood of symptomatic response and IBS-QOL score improvements (Figure 2). Referring patients with the most to gain from BHIs may not only maximize population-level improvements in QOL and disease experience but additionally could increase the overall value of outpatient gastroenterology services.

Finally, in the case of patients with IBD, functional complaints have been associated with higher healthcare utilization and costs. ${ }^{23}$ Psychiatric comorbidity has also been associated with hospital readmissions and unnecessary, costly diagnostic testing in this population. ${ }^{24,25}$ We found that behavioral health outcomes were not limited to disease, and therefore, it is important to consider patients with IBD for brain-gut psychotherapies despite limited research. ${ }^{6}$

Recognizing the positive impact on many digestive diseases, both gastroenterologists and patients are increasingly seeking

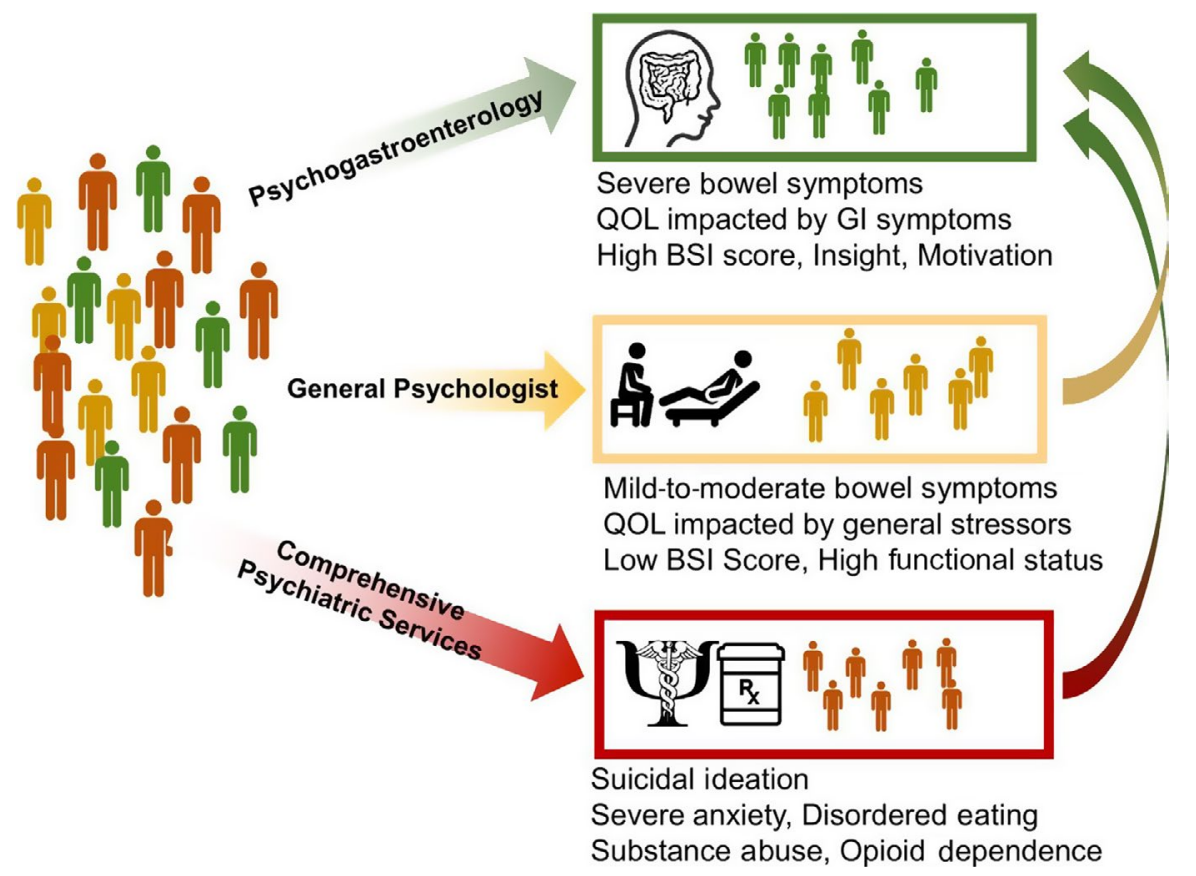

FIGURE 2 Proposed referral guide for mental and behavioral health services in patients with gastrointestinal conditions. The above schema incorporates predictors of improvement of quality of life in patients with gastrointestinal complaints following behavioral health interventions, as well as experience-based suggestions for most beneficial first mental health provider. Complex patients are encouraged to establish care with general mental health care OR establish comprehensive psychiatric care to stabilize mood. They may then present for re-assessment with a GI psychologist after 6 months of stable mood and treatment 
psychogastroenterology services. Incorporation of a validated complexity measure would be helpful in further developing a risk stratification model for the dissemination of GI behavioral health services. Future work will be aimed at evaluating economic factors such as changes in healthcare utilization and cost-effectiveness analyses when a more select group of GI patients are prioritized for behavioral health care. With improved pathways to care, our capacity to provide comprehensive care for digestive diseases will continue to improve.

\section{ACKNOWLEDGMENTS}

MER: study concept and design, analysis and interpretation of data, drafting and critical revision of manuscript; JAK: study concept and design, analysis and interpretation of data, drafting and critical revision of manuscript; WDC: analysis and interpretation of data, critical revision of the manuscript; RWS: study concept and design, statistical analysis, analysis and interpretation of data, drafting and critical revision of manuscript.

\section{DISCLOSURES}

The authors declare that there are no conflicts of interest.

\section{ORCID}

Megan E. Riehl (iD https://orcid.org/0000-0002-8219-9409

\section{REFERENCES}

1. Addolorato G, Mirijello A, D'Angelo C, et al. State and trait anxiety and depression in patients affected by gastrointestinal diseases: psychometric evaluation of 1641 patients referred to an internal medicine outpatient setting. Int J Clin Pract. 2008;62:1063-1069.

2. Gracie DJ, Guthrie EA, Hamlin PJ, Ford AC. Bi-directionality of brain-gut interactions in patients with inflammatory bowel disease. Gastroenterology. 2018;154(6):1635-1646.e3.

3. Ballou S, Keefer L. Psychological interventions for irritable bowel syndrome and inflammatory bowel diseases. Clin Transl Gastroenterol. 2017;8(1):e214.

4. Kinsinger SW. Cognitive-behavioral therapy for patients with irritable bowel syndrome: current insights. Psychol Res Behav Manag. 2017;10:231-237.

5. Riehl ME, Kinsinger S, Kahrilas PJ, Pandolfino JE, Keefer L. Role of a health psychologist in the management of functional esophageal complaints. Dis Esophagus. 2015;28:428-436.

6. Taft TH, Ballou S, Bedell A, Lincenberg D. Psychological considerations and interventions in inflammatory bowel disease patient care. Gastroenterol Clin North Am. 2017;46:847-858.

7. Yadlapati R, Tye M, Keefer L, Kahrilas PJ, Pandolfino JE. Psychosocial distress and quality of life impairment are associated with symptom severity in PPI Non-responders with normal impedance-pH profiles. Am J Gastroenterol. 2018;113:31-38.

8. Drossman DA, Patrick DL, Whitehead WE, et al. Further validation of the IBS-QOL: a disease-specific quality-of-life questionnaire. Am J Gastroenterol. 2000;95:999-1007.

9. Drossman $\mathrm{D}$, Morris $\mathrm{CB}, \mathrm{Hu} \mathrm{Y}$, et al. Characterization of health related quality of life (HRQOL) for patients with functional bowel disorder (FBD) and its response to treatment. Am J Gastroenterol. 2007;102:1442-1453.
10. Derogatis LR, Melisaratos N. The Brief Symptom Inventory: an introductory report. Psychol Med. 1983;13:595-605.

11. DeRogatis H. A different reflection. Nurs Outlook. 1993;41:235-237.

12. Gracie DJ, Irvine AJ, Sood R, Mikocka-Walus A, Hamlin PJ, Ford AC. Effect of psychological therapy on disease activity, psychological comorbidity, and quality of life in inflammatory bowel disease: a systematic review and meta-analysis. Lancet Gastroenterol Hepatol. 2017;2:189-199.

13. Peters SL, Muir JG, Gibson PR. Review article: gut-directed hypnotherapy in the management of irritable bowel syndrome and inflammatory bowel disease. Aliment Pharmacol Ther. 2015;41:1104-1115.

14. Palsson OS. Hypnosis treatment of gastrointestinal disorders: a comprehensive review of the empirical evidence. Am J Clin Hypn. 2015;58:134-158.

15. Laird KT, Tanner-Smith EE, Russell AC, Hollon SD, Walker LS. Comparative efficacy of psychological therapies for improving mental health and daily functioning in irritable bowel syndrome: a systematic review and meta-analysis. Clin Psychol Rev. 2017;51:142-152.

16. Palsson OS. Standardized hypnosis treatment for irritable bowel syndrome: the North Carolina protocol. Int J Clin Exp Hypn. 2006;54:51-64.

17. Keefer L, Taft TH, Kiebles JL, Martinovich Z, Barrett TA, Palsson OS. Gut-directed hypnotherapy significantly augments clinical remission in quiescent ulcerative colitis. Aliment Pharmacol Ther. 2013;38:761-771.

18. Riehl ME, Keefer L. Hypnotherapy for Esophageal Disorders. Am J Clin Hypn. 2015;58:22-33.

19. Kiebles JL, Kwiatek MA, Pandolfino JE, Kahrilas PJ, Keefer L. Do patients with globus sensation respond to hypnotically assisted relaxation therapy? A case series report. Dis Esophagus. 2010;23:545-553.

20. Gonsalkorale WM, Houghton LA, Whorwell PJ. Hypnotherapy in irritable bowel syndrome: a large-scale audit of a clinical service with examination of factors influencing responsiveness. Am J Gastroenterol. 2002;97:954-961.

21. Palsson OS, Turner MJ, Johnson DA, et al. Hypnosis treatment for severe irritable bowel syndrome: investigation of mechanism and effects on symptoms. Dig Dis Sci. 2002;47:2605-2614.

22. Palsson OS, Whitehead WE. Psychological treatments in functional gastrointestinal disorders: a primer for the gastroenterologist. Clin Gastroenterol Hepatol. 2013;11(3):208-216.

23. Gracie DJ, Hamlin PJ, Ford AC. Longitudinal impact of IBS-type symptoms on disease activity, healthcare utilization, psychological health, and quality of life in inflammatory bowel disease. Am J Gastroenterol. 2018;113:702-712.

24. Allegretti JR, Borges L, Lucci M, et al. Risk factors for rehospitalization within 90 days in patients with inflammatory bowel disease. Inflamm Bowel Dis. 2015;21:2583-2589.

25. Ananthakrishnan AN, Gainer VS, Perez RG, et al. Psychiatric comorbidity is associated with increased risk of surgery in Crohn's disease. Aliment Pharmacol Ther. 2013;37:445-454.

\section{SUPPORTING INFORMATION}

Additional supporting information may be found online in the Supporting Information section at the end of the article.

How to cite this article: Riehl ME, Kinnucan JA, Chey WD,

Stidham RW. Nuances of the psychogastroenterology patient: A predictive model for gastrointestinal quality of life improvement. Neurogastroenterol Motil. 2019;31:e13663. https://doi.org/10.1111/nmo.13663 